\title{
SINDROME GENITOURINARIO DE LA MENOPAUSIA: CLINICA Y MANEJO
}

Félix Dasio Ayala Peralta1 ${ }^{1}$ Dasio Ayala Moreno², Antonio Mambret Luna Figueroa ${ }^{3}$, César Carranza Asmat ${ }^{4}$, Emma Ysabel Quiñones Pereyra ${ }^{5}$, Richard Ayala Palomino ${ }^{6}$, Luis Alberto Quiñones Vásquez ${ }^{7}$, Jovanna Ochante Rementería ${ }^{8}$.

\begin{abstract}
RESUMEN
El síndrome genitourinario de la menopausia clínicamente se evidencia 4 a 5 años después de la menopausia, y los cambios objetivos, están presentes en un $25-57 \%$ de todas las mujeres postmenopáusicas. Los síntomas más frecuentes son sequedad vaginal $(75 \%)$, dispareunia (38\%), y prurito vaginal, flujo vaginal y dolor (15\%); y el diagnóstico se basa en criterios clínicos. Los principios terapéuticos están basados en la restauración de la fisiología urogenital y alivio de los síntomas; contando para ello, las opciones terapéuticas no-hormonales/lubricantes, terapia hormonal sistémica, terapia estrogénica local y otras alternativas. El síndrome genitourinario de la menopausia es uno de los determinantes más importantes de la función sexual y de la salud urogenital, con un impacto significativo en la calidad de vida relacionada con la salud femenina.
\end{abstract}

Palabras clave: Síndrome genitourinario de la menopausia; Atrofia vulvovaginal; Menopausia; Salud vaginal (Fuente: DeCS BIREME).

\section{GENITOURINARY SYNDROME OF MENOPAUSE: CLINIC AND MANAGEMENT}

\begin{abstract}
The genitourinary syndrome of menopause clinically is evident 4 to 5 years after menopause, and objective changes are present in $25-50 \%$ of all postmenopausal women. The most frequent symptoms are vaginal dryness ( $75 \%$ ), dyspareunia (38\%), and vaginal itching, vaginal discharge and pain (15\%); and the diagnosis is based on clinical criteria. The principles therapeutic are based on the restoration of urogenital physiology and relief of symptoms; including non-hormonal / lubricant therapeutic options, systemic hormonal therapy, local estrogen therapy and other alternatives. The genitourinary syndrome of menopause is one of the most important determinants of sexual function and urogenital health, with a significant impact on the quality of life related to female health.
\end{abstract}

Key words: Genitourinary syndrome of menopause; Vulvovaginal atrophy; Menopause; Vaginal health (Source: MeSH NLM).

\section{INTRODUCCCIÓN}

El tracto urogenital femenino es especialmente sensible a la disminución de los estrógenos, y aproximadamente la mitad de las mujeres postmenopáusicas experimentarán síntomas relacionados a la atrofia urogenital, afectando la función sexual y la calidad de vida relacionada con la salud.

El síndrome genitourinario de la menopausia (GSM) incluyen síntomas y signos asociados con la posmenopausia relacionadas a la deficiencia de estrógenos que implican cambios en los labios, vagina, uretra, vejiga e involucra atrofia vulvovaginal (VVA) ${ }^{1}$. Pueden presentar síntomas genitales de sequedad, ardor e irritación; síntomas sexuales de falta de lubricación, dispareunia y deterioro de la función sexual; y síntomas urinarios de urgencia, disuria e infección recurrente del tracto urinario ${ }^{2-4}$.

La sequedad vaginal es frecuente entre las mujeres de todas las edades; pero particularmente es común durante y después de la menopausia ${ }^{5}$.

Tasas de prevalencia reportadas de sequedad vaginal debido a GSM varían, pero se estima que aproximadamente el $15 \%$ de las mujeres premenopáusicas y hasta el $57 \%$ de las mujeres posmenopáusicas experimentan esta condición ${ }^{6}$.

La atrofia vulvovaginal (VVA) se aprecia clínicamente cuatro a cinco años después de la menopausia, y los

Médico GínecoObstetra. Magíster en Salud Reproductiva. Profesor de la Facultad de Medicina Humana de la Universidad San Martín de Porres y de Ciencias de la Salud de la Universidad Norbert Wiener. Jefe del Servicio de Climaterio y Menopausia del Instituto Nacional Materno Perinatal. Miembro de la Red Latinoamericana de Investigadores en Climaterio (REDLINC). Lima-Perú.

2 Médico Cirujano. Universidad Nacional Federico Villarreal, Lima-Perú.

3 Médico GínecoObstetra. Profesor Principal de la Universidad Nacional Mayor de San Marcos. Director Ejecutivo de Investigación, Docencia y Atención en Obstetricia y Ginecología del Instituto Nacional Materno Perinatal. Lima-Perú.

4 Médico GínecoObstetra. Profesor Asociado de la Universidad Nacional Mayor de San Marcos. Director Ejecutivo de la Oficina de Investigación y Docencia Especializada. Instituto Nacional Materno Perinatal. Lima-Perú.

Médico Cirujano. Universidad San Martín de Porres. Lima-Perú

Médico Cirujano. Universidad Privada San Juan Bautista. Lima-Perú.

Médico GínecoObstetra. Asistente del Servicio de Obstetricia C. Instituto Nacional Materno Perinatal. Lima-Perú

Licenciada en Trabajo Social. Servicio de Trabajo Social. Instituto Nacional Materno Perinatal. Lima-Perú.

$$
\text { Recibido: 14-01-17 Aprobado: 30-06-17 }
$$


cambios objetivos, además de síntomas subjetivas, están presentes en un $25-50 \%$ de todas las mujeres postmenopáusicas ${ }^{7}$.

Las concentraciones séricas de estradiol en las mujeres premenopáusicas oscilan entre los 147 y los $1468 \mathrm{pmol} / \mathrm{l}$ $(40-400 \mathrm{pg} / \mathrm{ml})$ y caen a menos de $73 \mathrm{pmol} / \mathrm{l}(20 \mathrm{pg} / \mathrm{ml})$ posterior a la menopausia ${ }^{8}$.

\section{FISIOLOGÍA VAGINAL EN LA POSTMENOPAUSIA}

La disminución de estrógenos circulantes asociada con la transición menopáusica se correlaciona estrechamente con: disminución de lactobacilos vaginales, aumento del $\mathrm{pH}$, alteración de la morfología epitelial, disminución del flujo vascular y reducción de las secreciones fluidas de la vagina ${ }^{7}$.

La pérdida de la producción ovárica de estrógenos se asocia con GSM. Este cambio en los estrógenos circulantes se refleja en la fisiología vaginal y en la aparición de síntomas (Figura 1). La vagina es un indicador biológico accesible y sensible a la disminución de los estrógenos; y de sus niveles circulantes bajos en mujeres postmenopáusicas ${ }^{7,8}$.

Los estrógenos promueven la formación de glicógeno en el epitelio escamoso. Los lactobacilos de Döderlein, parte de la flora vaginal normal, dependen del glicógeno como fuente metabólica $v$ convierten el alicóaeno de células vaginales descamadas en ácido láctico, manteniendo así un pH vaginal ácido: 3,5 - 4,5. El pH ácido sirve para disminuir la infección por patógenos. Los estrógenos también ayudan a mantener el grosor del epitelio poliestratificado escamoso vaginal, que le confiere su color rosado, pliegues y humedad normales. En ausencia de estrógenos, aumenta la proliferación del tejido conjuntivo, hay fragmentación de la elastina, y el colágeno se hialiniza ${ }^{7,8}$.

Las mujeres postmenopáusicas tienen un volumen estimado total de fluido vaginal de $0,0825 \mathrm{~g}$ por 15 -min de recolección, comparado con $0,214 \mathrm{~g}$ en mujeres fértiles. La mayor parte del fluido vaginal en mujeres postmenopáusicas sería secretado por el epitelio vaginal ${ }^{9}$.

$\mathrm{El} \mathrm{pH}$ vaginal en mujeres premenopáusicas es menor que 4,5, reflejo de la producción de ácido láctico por los lactobacilos vaginales. Este se eleva sobre 6 en mujeres postmenopáusicas debido a la disminución en la colonización de lactobacilos en la vagina, consecuencia de la reducción de células superficiales y por lo tanto de glicógeno, con un epitelio vaginal adelgazado ${ }^{7,8,10}$. En consecuencia, la vagina postmenopáusica está en riesgo de sufrir inflamación e infección ${ }^{10}$.

El hallazgo clínico de la pérdida de los pliegues rugosos vaginales y el adelgazamiento del epitelio se presentan a los 2-3 años de postmenopausia. La ausencia de rugosidades se debe a la pérdida del soporte de coláceno del enitelio vacinal. La renovación del colágeno

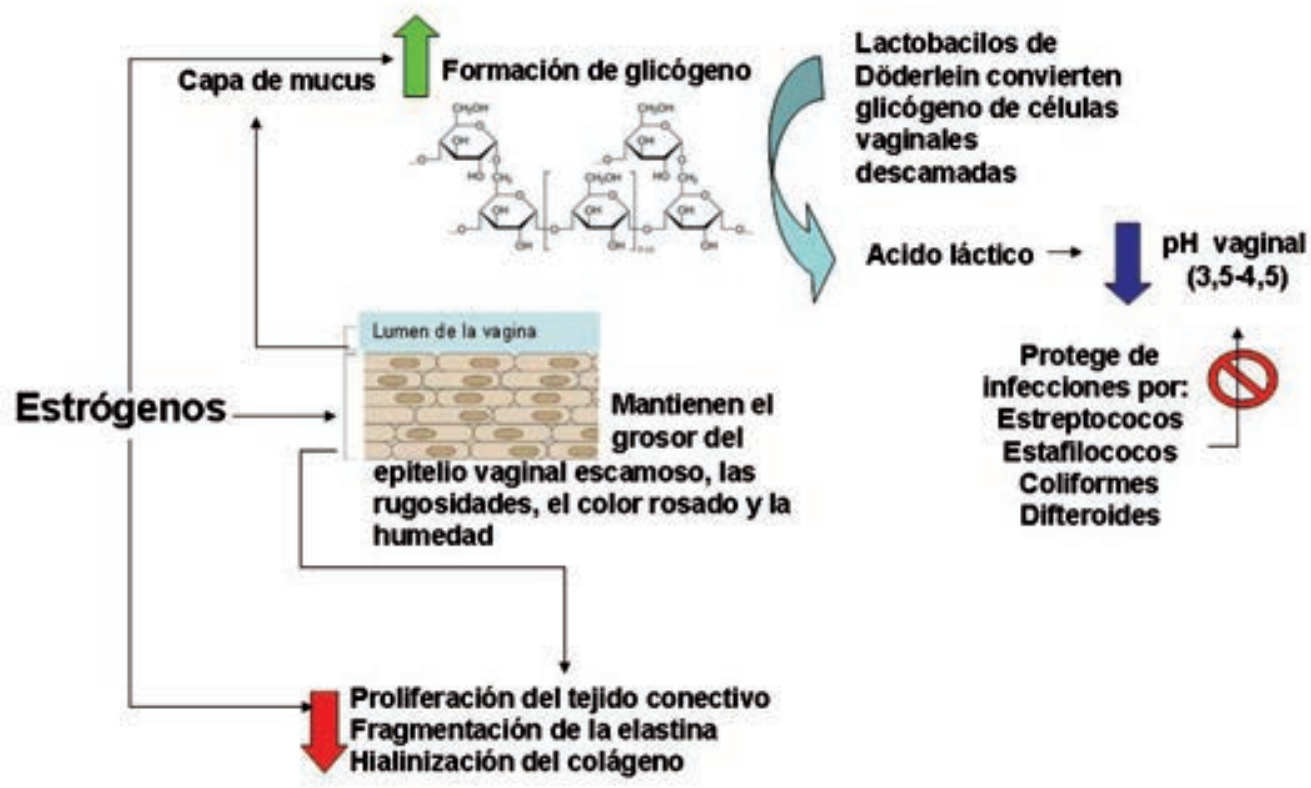

Figura 1. Efectos de los estrógenos en el epitelio vaginal en forma esquemática. Tomada de Sturdee DW, Panay N. International Menopause Society (IMS). Recommendations for the management of postmenopausal vaginal atrophy. Climateric 2010;Early Online,1-14. 
disminuye en las mujeres posmenopáusicas sin terapia hormonal, y estos cambios pueden ser importantes para el prolapso vaginal ${ }^{11}$. La sequedad vaginal ocurre temprano en el período postmenopáusico, y se manifiesta más en mujeres sexualmente activas con dispareunia ${ }^{7,8}$.

\section{SALUD VAGINAL Y FUNCION SEXUAL}

La salud vaginal juega un rol crucial en la salud sexual, y los estrógenos modulan el proceso hemodinámico involucrado en el ciclo de respuesta sexual. Cuando la atrofia vaginal es evidente, las mujeres menopáusicas pueden quejarse de sequedad vaginal y, cuando están sexualmente activas, pueden experimentar dolor con el acto sexual (dispareunia).

La atrofia vaginal es uno de los determinantes más importantes de la función sexual y de la salud urogenital, con un impacto significativo en la calidad de vida.

Las mujeres que experimentan síntomas sexuales y urinarios como consecuencia de la atrofia vaginal debieran ser diagnosticadas y tratadas a la brevedad para así evitar el caer en una cascada de eventos que no son posibles de resolverse espontáneamente. Sin embargo, la experiencia de síntomas sexuales es única en cada mujer, y ésta es influenciada no sólo por la edad y por la menopausia, sino también por una compleja interacción de factores personales que afectan la calidad de vida y la relación de pareja $a^{7,12}$.

Un estudio de REDLINC mostró que $28,7 \%$ de las mujeres premenopáusicas de 40 a 44 años de edad notaba disminución de la lubricación vaginal, porcentaje que se eleva a $37,5 \%$ en premenopáusicas de 45 o más años, a $49,8 \%$ en la perimenopáusicas ${ }^{13}$. Igualmente, los problemas sexuales y las molestias urinarias siguen un comportamiento similar. En las mujeres latinoamericanas la atrofia vaginal es una causa importante de sintomatología menopáusica, perjudicando su función sexual y su calidad de vida ${ }^{13}$

La Red Latinoamericana de Investigación en Climaterio $(\text { REDLINC) })^{14}$ analizó el Indice de Función Sexual Femenina (FSFI) en 7243 mujeres entre 40-59 años de edad en 11 países latinoamericanos y describió una alta prevalencia de disfunción sexual (56,8\%). EI FSFI evalúa diversos dominios de la función sexual: deseo, excitación, orgasmo, dolor, lubricación y satisfacción. El factor de riesgo más importante para la disfunción sexual en la población estudiada fue la sequedad vaginal (odds ratio 3,86, intervalo de confianza 95\% $3,37-4,43)^{14}$. Un estudio en mujeres nativas Movima de Bolivia mostró que los síntomas asociados con atrofia genital se encontraban entre las principales quejas de la menopausia: dispareunia (40\%), prurito genital $(40,8 \%)$ y pérdida de libido $(51 \%)^{15}$.

\section{CLÍNICA DEL SÍNDROME GENITOURINARIO DE LA MENOPAUSIA SINTOMAS PRINCIPALES EN GSM}

Los síntomas más frecuentes de GSM son sequedad (estimada en 75\%), dispareunia (estimada en 38\%), y prurito vaginal, flujo vaginal y dolor (estimados en 15\%). En la tabla 1 se mencionan los síntomas vulvares, vaginales y del tracto urinario ${ }^{7}$. Si bien puede cambiar la frecuencia relativa de los dos síntomas más comunes (dispareunia y sequedad), estos dos síntomas son generalmente los dos más usuales. La dispareunia puede afectar adversamente la calidad de la vida sexual de la mujer postmenopáusica, o intensificar afecciones sexuales pre-existentes ${ }^{7,16}$.

Tabla 1. Síntomas urogenitales, cambios fisiológicos y cambios anatómicos relacionados a la deficiencia de estrógenos

\section{Vulva}

- Pérdida de la almohadilla adiposa labial

- Retracción y pérdida de la definición de los labios mayores y menores

- Acortamiento del prepucio y exposición excesiva del clítoris

- Susceptibilidad a irritantes químicos y físicos, a traumas mecánicos e infecciones

- Pérdida del vello púbico

\section{Vagina}

- Sequedad, humedad insuficiente

- Flujo sanguíneo disminuido

- Dispareunia

- Prurito

- Sensación de ardor

- Dolor

- Pérdida de elasticidad

- Adelgazamiento del tejido vaginal y alteración de la queratinización

- Defectos en la mucosa incluyendo petequias, microfisuras, ulceración e inflamación

- Acortamiento, fibrosis, obliteración de la cúpula vaginal y/o estrechamiento del introito vaginal

- Alisamiento de los fondos de saco, aplanamiento de los pliegues/rugosidades vaginales

- Susceptibilidad a traumas mecánicos

- Impacto adverso en la curación de heridas traumáticas y postoperatorias

- Indice de maduración vaginal anormal: porcentaje disminuido de capas celulares superficiales, porcentaje aumentado de células parabasales

- Contenido de glicógeno disminuido en células epiteliales vaginales

- Expulsión de la flora facultativa de la vagina que mantiene contenidos a los micoorganismos patógenos

- Aumento del $\mathrm{pH}$ vaginal por sobre 5,0

- Leucorrea y/o secreción anormal

- Infiltración de la submucosa por linfocitos y células plasmáticas

Vejiga y uretra 
- Aumento del residuo vesical post-miccional

- Disminución de la capacidad vesical

- Disminución de la presión máxima de contracción miccional del músculo detrusor de la vejiga

- Disminución del umbral de sensibilidad a la distensión de la vejiga (sensación primera de urgencia)

- Disminución de la presión de cierre uretral

- Disminución de la perfusión de los plexos venosos periuretrales

- Disminución del flujo de orina uretral

- Indice de maduración uretral anormal: porcentaje disminuido de capas celulares superficiales, porcentaje aumentado de células parabasales

- Síntomas de disuria, nicturia y urgencia

- Incontinencia urinaria

- Infecciones urinarias recurrentes

- Alteración en la biosíntesis de colágeno en el tejido conjuntivo periuretral

Referencia: Tomada de Sturdee DW, Panay N. International Menopause Society (IMS). Recommendations for the management of postmenopausal vaginal atrophy. Climateric 2010; Early Online, 1-14.

\section{SIGNOS FÍSICOS EN GSM}

Los signos físicos en GSM presentes son: disminución de las rugosidades vaginales y reducción del flujo sanguíneo, resultando en una apariencia pálida; cambio en el $\mathrm{pH}$ vaginal desde un rango moderadamente ácido $(\mathrm{pH} 3,5$ $5,0)$ a un rango neutro $(\mathrm{pH} 6,0-8,0)$; y un cambio en el índice de maduración vaginal ${ }^{7}$.

Con la declinación de los estrógenos se adelgazan la mucosa del cérvix uterino, y de los epitelios vaginal y vulvar, haciéndose más susceptibles de lesionarse. Las rugosidades vaginales disminuyen, llevando a una pared vaginal de apariencia lisa, que se acompaña de una disminución en el flujo sanguíneo. Combinados, estos cambios resultan en una vagina de aspecto pálido y con la eventual presencia de petequias pequeñas y/u otros signos de inflamación. Mientras que la acidez fisiológica de la vagina estrogenizada está dentro del rango moderadamente ácido (rango normal: $\mathrm{pH} 3,5-5,0$, favoreciendo los lactobacilos), el $\mathrm{pH}$ se eleva con la declinación de los estrógenos (rango de $\mathrm{pH}$ entre 6,0-8,0, favoreciendo organismos patógenos que incluyen levaduras y bacterias, como las coliformes). Este $\mathrm{pH}$ más alcalino lleva a un cambio en la flora vaginal hacia el aumento de coliformes que, junto a los otros cambios atróficos, son responsables del aumento en la frecuencia y susceptibilidad de infecciones y mal olor vaginal ${ }^{17}$, como también de sangrado por trauma asociado al acto sexual o a la inserción del espéculo en el examen ginecológico rutinario. Pueden observarse úlceras micro o macroscópicas en el epitelio vaginal, ya sea espontáneamente o con un trauma menor. En pacientes sexualmente inactivas y en aquéllas con actividad coital esporádica, la atrofia vaginal severa puede resultar en un estrechamiento, acortamiento e incluso obliteración de la vagina ${ }^{18}$.
Los cambios anatómicos y fisiológicos del tracto urinario relacionados a la deficiencia estrogénica pueden también causar o acentuar una disfunción de la micción, incluyendo aumento en la frecuencia urinaria, disuria, nicturia, como también urgencia miccional, incontinencia urinaria de urgencia o formas mixtas de incontinencia ${ }^{18}$. La deficiencia estrogénica causa cambios atróficos en el trígono vesical, disminución de la tensión de la musculatura y de las estructuras conectivas del diafragma urogenital, alteraciones del metabolismo del colágeno y disminución de la actividad del sistema $\alpha$-adrenérgico que inerva tanto el cuello vesical como el esfínter uretral ${ }^{7}$. La mucosa uretral, también estrógeno-sensible, se adelgaza junto con su plexo vascular submucoso. En conjunto, estos cambios contribuyen a una disminución de la presión intrauretral, importante mecanismo que facilita la continencia urinaria, favoreciéndose entonces la pérdida de orina. Los mismos cambios vaginales del $\mathrm{pH}$ y de la flora bacteriana pueden tener un impacto en el tracto urinario inferior, aumentando el riesgo de uretritis y cistitis agudas y recurrentes ${ }^{7}$.

\section{DIAGNÓSTICO CLÍNICO DEL GSM}

El diagnóstico del GSM se basa en criterios clínicos, orientada hacia la presencia de síntomas genitourinarios, incluida la incontinencia urinaria de urgencia y un aumento de infecciones del tracto urinario, y en la exploración clínica, que revelará una disminución de los pliegues vaginales o adelgazamiento de la mucosa y afectación de la piel de la vulva ${ }^{19}$.

Históricamente, las dos principales mediciones objetivas, tanto para diagnóstico como para evaluación de eficacia terapéutica, han sido el $\mathrm{pH}$ vaginal, y el índice de maduración vaginal (IMV). EI IMV corresponde al cálculo de los porcentajes relativos de las células superficiales comparado con las células intermedias y parabasales ${ }^{7}$.

Actualmente, se incluyen escalas de resultados autoreportados por pacientes como parte de la evaluación. Las pacientes sintomáticas hacen una selección de sus síntomas más molestos (sequedad vaginal, dispareunia, irritación vaginal, disuria, dolor vaginal, sangrado postcoital) y estiman su intensidad en una escala de tres o cuatro puntos. Un beneficio terapéutico documentado consiste en una mejoría estadísticamente significativa en tres objetivos co-primarios: $\mathrm{pH}$ vaginal, IMV y el síntoma más molesto, al comparar con individuos tratados con placebo ${ }^{7}$.

\section{TRATAMIENTO DEL SINDROME GENITOURINARIO DE LA MENOPAUSIA}

Los principios terapéuticos en mujeres con GSM establecida son: a) restauración de la fisiología urogenital [la terapia estrogénica disminuye el $\mathrm{pH}$ vaginal, engruesa el epitelio, aumenta el flujo sanguíneo y mejora la 
lubricación vaginal]; y b) alivio de los síntomas [la restauración de la fisiología urogenital lleva al alivio de varios síntomas vaginales tales como sequedad vaginal, dispareunia superficial y profunda, vulvodinia, sangrado vulvovaginal, inflamación y flujo vaginal] ${ }^{7}$.

Tenemos las siguientes opciones terapéuticas:

a) Tratamientos no-hormonales/lubricantes

b) Terapia hormonal sistémica

c) Terapia estrogénica local

d) Otras alternativas

\section{Tratamiento No-hormonales/lubricantes}

La terapia de primera línea para la mujer con atrofia vulvovaginal (VVA) sintomática debe incluir lubricantes no hormonales durante el coito $\mathrm{y}$, uso regular de cremas hidratantes vaginales de acción prolongada [Nivel A].

Las opciones no-hormonales se indican fundamentalmente en mujeres que desean evitar la terapia hormonal o en aquéllas de alto riesgo con historia de neoplasia maligna sensible a hormonas, como cáncer mamas o de endometrio.

Consisten principalmente en una combinación de agentes protectores y espesantes en una base soluble en agua. Los lubricantes son utilizados principalmente para aliviar la sequedad vaginal durante el acto sexual.

En un ensayo clínico reciente de un hidratante vaginal comparado con estrógenos vaginales en baja dosis; ambos preparados estrogénicos vaginales de baja dosis fueron efectivos sobre los síntomas y salud vaginal, mientras que el hidratante no-hormonal otorgó solamente beneficios transitorios ${ }^{20}$.

Preparados de fitoestrógenos. Existen datos que demuestran beneficios en la actividad urogenital de preparados fitoestrogénicos, tales como isoflavonas de soya y trébol rosado ${ }^{20} .7$ Ocho semanas de $40 \mathrm{mg}$ de isoflavonas de trébol rosado disminuyeron las células parabasales y aumentaron las células superficiales, aumentando así el índice de maduración vaginal sin efecto significativo en el grosor endometrial ${ }^{7}$.

Otros productos. Terapias complementarias tales como ortiga, raíz de consuelda (comfrey root), raíz de dong quai, agripalma (motherwort), ñame silvestre (wild yam), cápsulas de acidófilus y de brionia no han probado eficacia ni seguridad en ensayos clínicos controlados aleatorios ${ }^{21}$.

\section{Terapia hormonal sistémica}

La administración de estrógenos exógenos restaura el $\mathrm{pH}$ vaginal normal, engruesa y revasculariza el epitelio, y aumenta la lubricación vaginal. Como resultado, la terapia hormonal $(\mathrm{TH})$ alivia los síntomas relacionados a la atrofia vaginal, que incluyen sequedad, irritación, prurito, dispareunia y urgencia urinaria, y también puede disminuir la incidencia de infecciones urinarias bajas ${ }^{7}$.

La TH sistémica alivia la atrofia vaginal en alrededor de $75 \%$ de las mujeres. Por lo tanto, un $10-25 \%$ de las mujeres utilizando terapia hormonal sistémica seguirán experimentando sintomatología de atrofia urogenital. Este hallazgo, además de las aprehensiones de seguridad de la TH oral/transdérmica, son razones para no recomendar en forma usual una terapia sistémica en mujeres con síntomas vaginales exclusivos ${ }^{22}$.

La combinación de terapia sistémica y local puede ser necesaria inicialmente en algunas mujeres.

\section{Terapia estrogénica local}

La terapia estrogénica local puede ser administrada en tabletas, óvulos, cremas o un anillo vaginal. Esta terapia está disponible como estrógenos conjugados de equino, estradiol, estriol o estrona ${ }^{\text {? }}$.

Los estrógenos son fácilmente absorbidos en la pared vaginal y sus efectos no serán exclusivamente locales a menos que se usen formulaciones farmacéuticas que prevengan la absorción. Aún así existe cierta absorción, especialmente durante el inicio del tratamiento, cuando el epitelio vaginal aún es atrófico. La absorción disminuye cuando el epitelio madura como resultado de la terapia $y$, además, se requieren menores dosis de estrógenos para prevenir la recurrencia de la atrofia. Se necesitan habitualmente sólo dosis bajas para tratar síntomas vaginales en comparación con los síntomas sistémicos, y se pueden usar estrógenos de baja potencia como estriol, que otorgan un efecto suficiente en la vagina y con efectos sistémicos limitados a pesar de su absorción.

La tableta de estradiol de $25 \mu \mathrm{g}$ induce concentraciones estacionarias de estradiol de $5-10 \mathrm{pg} / \mathrm{ml}$, mientras que con la tableta de $10 \mu \mathrm{g}$, los niveles séricos de estradiol en estado estacionario no superan los $5 \mathrm{pg} / \mathrm{ml}$, aún así siendo más efectiva que placebo ${ }^{23}$.

Numerosos estudios clínicos y una revisión de Cochrane han evaluado la necesidad del uso concurrente de una progestina en mujeres en tratamiento con estrógenos vaginales. Los preparados estudiados incluyen estriol en crema y óvulos, estradiol en tabletas vaginales en dos dosis: $25 \mu \mathrm{g}$ y $10 \mu \mathrm{g}$, crema de estrógenos equinos conjugados (ECE) en dos dosis, y anillos vaginales impregnados de estradiol. Si bien los preparados de estriol no parecen estimular el endometrio, los estrógenos conjugados y preparados de estradiol sí podrían hacerlo en forma dosis-dependiente ${ }^{24}$

En un estudio reciente con crema de ECE en dosis baja $(0,3 \mathrm{mg})$ se reportó un endometrio proliferativo en 6 de 423 
mujeres con un seguimiento de más de 52 semanas, y no hubo casos de hiperplasia ni de carcinoma ${ }^{25}$.

La incidencia de hiperplasia observada en estos estudios es muy baja y similar a la observada en la población postmenopáusica no tratada. Una revisión de estrógenos tópicos del 2009 concluyó que ningún estudio evidencia proliferación endometrial luego de 6-24 meses de uso ${ }^{26}$, de manera que la literatura proporciona tranquilidad respecto a la seguridad de los preparados estrogénicos vaginales de baja dosis y no apoya el uso concomitante de progestinas sistémicas para la protección endometrial.

Esta evidencia ha sido apoyada en las guías de práctica clínica recientes de la Sociedad Norteamericana de Menopausia $(\mathrm{NAMS})^{27}$ y de la Sociedad Internacional de Menopausia (IMS) ${ }^{28}$,en que ninguna de estas organizaciones aboga el uso de progestinas en mujeres que utilizan preparados estrogénicos tópicos en forma apropiada.

\section{TIPOS DE ESTRÓGENO VAGINAL.}

Para las mujeres sintomáticas con moderada a severa VVA y para aquellas con VVA leves que no responden a lubricantes y humectantes, la terapia de estrógenos por vía vaginal en dosis bajas o sistémica sigue siendo la terapéutica estándar. Se prefiere estrógenos vaginales en dosis bajas cuando la VVA es el único síntoma de la menopausia[Nivel A] ${ }^{29}$.

El uso del producto con estrógeno vaginal para el tratamiento del GSM sintomático y vaginitis atrófica está aprobado en los Estados Unidos y Canadá (Tabla 1) ${ }^{29}$. Un producto con estrógeno vaginal es el anillo de acetato de estradiol (Femring) que suministra una dosis sistémica de estrógenos. Este producto está aprobado para el tratamiento de los síntomas vasomotores, además de la atrofia vulvovaginal. Femring no debe confundirse con Estring, que ofrece una dosis baja de estrógeno y está indicado solo para atrofia vulvovaginal ${ }^{29,30}$.

El tratamiento con estrógeno en cremas o comprimidos debe ser individualizada. Cuando se alcanza una respuesta terapéutica, típicamente después de 2 semanas de uso diario, la frecuencia de uso puede ser a menudo reducido. Un programa de mantenimiento de 2 a 3 dosis por semana es común, pero la dosis debe ajustarse a la dosis más baja y la frecuencia de estrógeno vaginal que proporciona el efecto deseado.

Aunque 2 dosis de la tableta vaginal muestra ser efectiva como es recomendable, pero se prefiere, la dosis más baja $(10 \mu \mathrm{g})$ y actualmente es la única dosis disponible en el Estados Unidos y Canadá ${ }^{29}$. Después de 2 semanas de dosificación diaria, la mujer puede utilizar dosis estándar de mantenimiento dos veces por semana o si prefiere con menor frecuencia.
Tabla 1. Terapia estrogénica $(E T)$ vaginal disponible para el uso en la posmenopausia ${ }^{29}$

\begin{tabular}{|c|c|c|}
\hline Composición & Producto & Dosis aprobada por FDA \\
\hline \multicolumn{3}{|c|}{ Cremas vaginales } \\
\hline $17 \beta$-estradiol & $\begin{array}{l}\text { Estrace } \\
\text { Vaginal } \\
\text { Creama }\end{array}$ & $\begin{array}{l}\text { Al inicio: } 2-4 \mathrm{~g} / \mathrm{d} \text { por } 1-2 \text { semana } \\
\text { Mantenimiento: } 1 \mathrm{~g} / 1-3 \quad \text { veces/ } \\
\text { semanac }^{\mathrm{c}} \\
(0,1 \mathrm{mg} \text { ingrediente activo/g) }\end{array}$ \\
\hline $\begin{array}{l}\text { Estrógeno } \\
\text { conjugado }\end{array}$ & $\begin{array}{l}\text { Premarin } \\
\text { Vaginal } \\
\text { Cream }^{d}\end{array}$ & $\begin{array}{l}\text { Para VVA: } 0,5-2 \mathrm{~g} / \mathrm{d} \text { por } 21 \mathrm{~d} \\
\text { seguido de } 7 \mathrm{~d}^{\mathrm{c}} \text {. } \\
\text { Para dispareunia: } 0,5 \mathrm{~g} / \mathrm{d} \text { por } 21 \mathrm{~d} \\
\text { seguido de } 7 \mathrm{~d} \text {, o dos veces } / \mathrm{sem}^{\mathrm{c}} \\
(0,625 \mathrm{mg} \text { ingrediente activo/g) }\end{array}$ \\
\hline Estrone & $\begin{array}{l}\text { Estragyn } \\
\text { Vaginal } \\
\text { Cream }^{\mathrm{b}}\end{array}$ & $\begin{array}{l}2-4 \mathrm{~g} / \mathrm{d} \text { (1 mg ingrediente activo/g) } \\
\text { Previsto para el uso a corto plazo; } \\
\text { se recomienda progestágeno. }\end{array}$ \\
\hline \multicolumn{3}{|c|}{ Anillos Vaginales } \\
\hline $\begin{array}{l}\text { Acetato de } \\
17 \beta \text {-estradiol }\end{array}$ & $\begin{array}{l}\text { Estring } \\
\text { Femring }^{\mathrm{a}}\end{array}$ & $\begin{array}{l}\text { Dispositivo que contiene } \\
\text { aproximadamente } 2 \mathrm{mg} \text { con liberación } \\
7,5 \mu \mathrm{g} / \mathrm{d} \text { por } 90 \mathrm{~d} \text { (para WVA) } \\
\text { Dispositivo que contiene } 12,4 \mathrm{mg} \\
\text { o } 24,8 \mathrm{mg} \text { de acetato de estradiol } \\
\text { con liberación } 0,05 \mathrm{mg} / \mathrm{d} \text { o } 0,10 \\
\mathrm{mg} / \mathrm{d} \text { estradiol por } 90 \text { días (ambas } \\
\text { dosis liberan niveles sistémicos } \\
\text { para el tratamiento de los síntomas } \\
\text { vasomotores y VVA) }\end{array}$ \\
\hline \multicolumn{3}{|c|}{ Tableta Vaginal } \\
\hline $\begin{array}{l}\text { Estradiol } \\
\text { hemihydrate }\end{array}$ & Vagifem & $\begin{array}{l}\text { Inicial: } 1 \text { tableta/d por } 2 \text { sem } \\
\text { Mantenimiento: } 1 \text { tablet } 2 \text { veces/sem } \\
\text { (tableta contiene } 10,3 \mu \mathrm{g} \text { de } \\
\text { hemihidrato de estradiol, equivalente } \\
\text { a } 10 \mu \mathrm{g} \text { de estradiol; para VVA) }\end{array}$ \\
\hline
\end{tabular}

Abreviaciones: ET, terapia con estrógeno; FDA, Departamento de Control de Alimentos y Medicamentos de los EEUU; VVA, atrofia vulvovaginal.

a Disponible en los Estados Unidos, pero no Canadá.

6 Disponible en Canadá, pero no en los Estados Unidos.

Algunas dosis aprobadas por la FDA de los estrógenos conjugados y cremas de estradiol son mayores que los que se utilizan actualmente en la práctica clínica que han demostrado ser eficaces.

d Disponible en Perú.

Las dosis de 0,5 a $1 \mathrm{~g}$ de crema vaginal de estrógeno, que se utiliza 1-2 veces a la semana puede ser suficiente para muchas mujeres

El anillo vaginal de estradiol de liberación sostenida proporciona hasta 90 días de tratamiento continuo, una característica que atrae a muchas mujeres. Alivio eficaz de GSM ha sido documentado sistemáticamente en ensayos aleatorizados y controlados con este sistema de administración de estrógenos ${ }^{7}$.

\section{DURACIÓN, MONITOREO Y EVENTOS ADVERSOS DE LA TERAPIA}

En la actualidad no hay indicaciones relativas a la duración de la terapia. La única recomendación es que, si se va a implementar una terapia a largo plazo, se deben usar dosis bajas. Invariablemente, las mujeres obtendrán alivio 
sustancial de sus síntomas luego de alrededor de tres semanas de tratamiento, si bien en algunas mujeres se puede requerir 4-6 semanas antes de apreciar una mejoría adecuada. Alrededor del $80-90 \%$ de las mujeres logrará mejoría subjetiva, y el fracaso del tratamiento obliga a una re-evaluación para excluir otras condiciones subyacentes, tales como dermatitis/dermatosis o vulvodinia.

\section{EFECTOS ADVERSOS DE LA TERAPIA ESTROGÉNICA LOCAL}

Efectos adversos serios son particularmente poco comunes. Sin embargo, todos los preparados pueden asociarse a eventos adversos menores y pueden causar irritación o prurito vaginal, descarga vaginal, sangrado vaginal, dolor pelviano, sensibilidad mamaria y parestesias. La ocurrencia de estos eventos varía dependiendo de los preparados utilizados y, en general, parece que las cremas pueden asociarse a más de estos eventos que las tabletas y el anillo. Esto puede deberse al preparado en sí, a una mayor absorción o al uso inadvertido de dosis más altas que las recomendadas al ser administradas en la vagina ${ }^{31}$.

En la actualidad, no hay motivos para que las mujeres con atrofia vulvovaginal sintomática no utilicen terapia estrogénica local de baja dosis, por el tiempo que sea necesario según la presencia de síntomas. No obstante, es prudente investigar en forma completa a aquéllas pacientes que se presenten con cualquier tipo de sangrado vaginal para descartar patología endometrial ${ }^{7}$.

\section{OTRAS ALTERNATIVAS}

\section{Ospemifeno}

Es el único SERM aprobado en los Estados Unidos para el tratamiento de moderada a severa dispareunia. Es un SERM con efectos únicos vaginales. Dos estudios de 12 semanas de duración con el uso diario de ospemifeno 60 mg por vía oral mostraron mejoría en índice de maduración vaginal, $\mathrm{pH}$ vaginal, y en la mayoría de las molestias de la sequedad vaginal ${ }^{32,33}$.

\section{Lasofoxifeno}

Un ensayo aleatorizado y controlado de 387 mujeres posmenopáusicas con atrofia vulvovaginal reportaron reducción de los síntomas asociados con las relaciones sexuales ${ }^{34}$.

\section{DHEA intravaginal}

La dehidroepiandrosterona (DHEA) a 2 dosis de $3,25 \mathrm{mg}$ y $13 \mathrm{mg}$, una vez al día durante 12 semanas de ensayo mostraron mejorías en índice de maduración vaginal y el $\mathrm{pH}$ vaginal mejorando la sintomatología vaginal ${ }^{35}$.

\section{Sistema láser CO2 fraccionado}

Basados en los datos publicados hasta la fecha, las primeras experiencias con estudios pilotos a corto y medio plazo, muestran que el tratamiento mediante laser fraccionado de $\mathrm{CO} 2$, con una adecuada selección de pacientes, constituye una nueva opción terapéutica no hormonal para las mujeres postmenopáusicas con SGM, factible, eficaz y muy bien tolerada ${ }^{36-38}$.

Estudios recientes han demostrado que el láser fraccionado es una opción terapéutica no hormonal efectiva, sencilla, bien tolerada y sin efectos adversos, para el tratamiento de GSM. La reacción térmica originada produce restauración del epitelio vaginal, un proceso de neocolagénesis y una mayor vascularización que favorece la llegada de nutrientes, reestableciéndose la estructura de la mucosa, su grosor y trofismo funcional, mejorando por tanto la sintomatología ${ }^{39}$.

\section{DECLARACIÓN DE LA SOCIEDAD NORTEAMERICANA DE MENOPAUSIA CON RELACIÓN AL MANEJO DEL SINDROME GENITOURINARIA DE LA MENOPAUSIA ${ }^{40}$}

a. Las preparaciones de estrógenos vaginales de bajas dosis son efectivas y generalmente seguras para el tratamiento de GSM, con una absorción sistémica mínima, y se prefiere sobre las terapias sistémicas cuando ET se considera sólo para GSM.

b. Para las mujeres con cáncer de mama, la baja dosis de estrógeno vaginal debe ser considerada y prescrito en consulta con sus oncólogos.

c. La terapia con progestágeno no es necesaria con la ET vaginal a baja dosis; y el sangrado posmenopáusica en las mujeres que usan dosis baja de ET vaginal deberá contar con evaluación completa que incluyan ecografía transvaginal y/o biopsia endometrial.

d. Terapias sin prescripción de estrógenos que mejoran GSM en mujeres posmenopáusicas incluyen ospemifeno y DHEA intravaginal.

\section{CONCLUSIONES}

El GSM es una causa frecuente de síntomas molestos en la postmenopausia, causados por la deficiencia de estrógenos, cuyo manejo con baja dosis de ET vaginal es considerada como primera línea de tratamiento médico que puede transformar la calidad de vida de una mujer.

Financiamiento: autofinanciado.

Conflicto de interés: los autores declaran no tener algún conflicto de interés.

\section{REFERENCIAS BIBLIOGRÁFICAS}

1. Portman DJ, Gass ML. Vulvovaginal Atrophy Terminology Consensus Conference Panel. Genitourinary syndrome of menopause: new terminology for vulvovaginal atrophy from the International Society for the Study of Women's Sexual Health and the North American Menopause Society. Menopause 2014;21:1063-1068. 
2. The North American Menopause Society. Position Statement The 2017 Hormone Therapy Position Statement Of The North American Menopause Society. Menopause. 2017; 24( 7):1-26

3. Panay N. Genitourinary syndrome of the menopause -dawn of a new era. Climacteric 2015;18(Suppl 1):13-17

4. Kim HK, Kang SY, Chung YJ, Kim JH, Kim MR. The Recent Review of the Genitourinary Syndrome of Menopause. J Menopausal Med. 2015 Aug;21(2):65-71. doi: 10.6118/ jmm.2015.21.2.65. Epub 2015 Aug 28.

5. Panay N, Fenton A. Vulvovaginal atrophy-a tale of neglect. Climacteric 2014;17:1-2

6. Palacios S. Managing urogenital atrophy. Maturitas 2009;63:315-18

7. Sturdee DW, Panay N. International Menopause Society (IMS). Recommendations for the management of postmenopausal vaginal atrophy. Climateric 2010;Early Online,1-14.

8. Archer DF. Efficacy and tolerability of local estrogen therapy for urogenital atrophy. Menopause 2010;17:194-203.

9. Semmens JP, Wagner G. Estrogen deprivation and vaginal function in postmenopausal women. JAMA 1982;248:445-8.

10. Heinemann C, Reid G. Vaginal microbial diversity among postmenopausal women with and without hormone replacement therapy. Can J Microbiol 2005;51:777-81.

11. Tinelli A, Malvasi A, Rahimi S, et al. Age-related pelvic floor modifications and prolapse risk factors in postmenopausal women. Menopause 2010;17:204-12

12. Nappi RE, Lachowsky M. Menopause and sexuality: prevalence of symptoms and impact on quality of life. Maturitas 2009;63:138-41

13. Blümel JE, Chedraui P, Baron G, Belzares E, Bencosme A, Calle A, et al; Collaborative Group for Research of the Climacteric in Latin America (REDLINC). Menopausal symptoms appear before the menopause and persist 5 years beyond: a detailed analysis of a multinational study. Climacteric 2012; 15:542-51.

14. Blümel JE, Chedraui P, Baron G, et al. Collaborative Group for Research of the Climacteric in Latin America (REDLINC). Sexual dysfunction in middle-aged women: a multicenter Latin American study using the Female Sexual Function Index. Menopause 2009;16:1139-48

15. Castelo-Branco C, Palacios S, Mostajo D, et al. Menopausal transition in Movima women, a Bolivian native-American. Maturitas 2005;51:380-5

16. Bachmann GA, Leiblum SR, Kemmann E, et al. Sexual expression and its determinants in the post-menopausal woman. Maturitas 1984;6:19-29

17. Caillouette JC, Sharp CF Jr, Zimmerman GJ, Roy S. Vaginal pH as a marker for bacterial pathogens and menopausal status. Am J Obstet Gynecol 1997;176:1270-5

18. Robinson D, Cardozo L. The menopause and HRT. Urogenital effects of hormone therapy. Best Pract Res Clin Endocrinol Metab 2003;17:91-104

19. 13. Palacios S, Cancelo MJ. Guía práctica de asistencia del síndrome urogenital de la menopausia (SEGO). Actualización 2015. Ed: SEGO (junio 2015). Madrid.España.

20. Biglia N, Peano E, Sgandurra $P$, et al. Low-dose vaginal estrogens or vaginal moistuizer in breast cancer survivors with urogenital atrophy: a preliminary study. Gynecol Endocrinol 2010;26:404-12

21. Castelo-Branco C, Cancelo M, Villero J, Nohales F, Julia M. Management of postmenopausal vaginal atrophy and atrophic vaginitis. Maturitas 2005;52(Suppl 1):S46-52

22. Goldstein I. Recognizing and treating urogenital atrophy in postmenopausal women. J Womens Health (Larchmt) 2010;19:425-32

23. Eugster-Hausmann M, Waitzinger J, Lehnick D. Minimized estradiol absorption with ultra-low-dose $10 \mu \mathrm{g}$ 17ß-estradiol vaginal tablets. Climacteric 2010;13:219-27
24. Suckling J, Kennedy R, Lethaby A, Roberts H. Local oestrogen therapy for vaginal atrophy in post menopausal women. Cochrane Database Syst Rev 2006 Issue 4 CD 001500.

25. Bachmann G, Bouchard C, Hoppe D, et al. Efficacy and safety of low dose regimens of conjugated estrogen cream administered vaginally. Menopause 2009;16:719-27 .

26. Al-Baghdadi O, Ewies AAA. Topical estrogen therapy in the management of postmenopausal vaginal atrophy: an up-to-date overview. Climacteric 2009;12:91-105.

27. The North American Menopause Society. Estrogen and progestogen use in postmenopausal women; 2010 statement of the North American Menopause Society. Menopause 2010;17:242-55.

28. Pines A, Sturdee DW, Birkhauser MH, et al. IMS Updated Recommendations on postmenopausal hormone therapy. Climacteric 2007;10:181-94.

29. The North American Menopause Society. Management of symptomatic vulvovaginal atrophy: 2013 position statement of The North American Menopause Society. Menopause 2013;20(9):886-904

30. Bachmann G, Bouchard C, Hoppe D, et al. Efficacy and safety of low dose regimens of conjugated estrogens cream administered vaginally. Menopause 2009;16:719-727.

31. The North American Menopause Society. The role of local vaginal estrogen for treatment of vaginal atrophy in postmenopausal women: 2007 position statement of The North American Menopause Society. Menopause 2007;14:357-69

32. PortmanDJ, Bachmann GA, Simon JA; Ospemifene Study Group. Ospemifene, a novel selective estrogen receptor modulator for treating dyspareunia associated with postmenopausal vulvar and vaginal atrophy. Menopause 2013;20:623-630.

33. Bachmann GA, Komi JO; Ospemifene Study Group. Ospemifene effectively treats vulvovaginal atrophy in postmenopausal women: results from a pivotal phase 3 study. Menopause 2010;17:480-486.

34. Bachmann G, Gass M, Kagan R, Moffett A, Barcomb L, Symons J. Lasofoxifene (LASO), a next generation selective estrogen response modulator (SERM) improves dyspareunia in postmenopausal women with vaginal atrophy (VA) [abstract]. Menopause 2005;12:238.

35. Labrie F, Archer D, Bouchard C, et al. High internal consistency and efficacy of intravaginal DHEA for vaginal atrophy. Gynecol Endocrinol 2010;26:524-532.

36. Salvatore S, Nappi RE, Zerbinati N, et al. A 12-week treatment with fractional CO2 laser for vulvovaginal atrophy: a pilot study. Climateric 2014;17:363-9.

37. Perino A, Calligaro A, Forlani F, et al. Vulvo-vaginal atrophy: a new treatment modality using thermo-ablative fractional $\mathrm{CO} 2$ laser. Maturitas 2015;80:296-301.

38. Gambacciani M, Levancini M, Cervigni M. Vaginal erbium laser: the second-generation thermotherapy for the genitourinary syndrome of menopause. Climateric 2015;18:1-7.

39. Escribano JJ, Rodea G, Martín A, Cristobal I, Gonzáles PJ, Salinas J, Sánchez-Borrego R. Tratamiento del síndrome genitourinario de la menopausia mediante láser fraccionado CO2: una opción terapéutica emergente. Rev Chil Obstet Ginecol 2016; 81(2): 138 - 151

40. The North American Menopause Society. The 2017 hormone therapy position statement of the North American Menopause Society. Menopause 2017;24(7):1-26.

\section{Correspondencia:}

Dirección: Jirón Maracaibo 2153- San Martín de Porres-Lima 31.

Teléfono: 567-6501 Celular: 999227657

E-mail: fayala1401@hotmail.com 